\title{
Reação de germoplasma e híbridos de mamoeiro à mancha- de-phoma (Phoma caricae-papayae) em condições de campo
}

\author{
Marcelo Vivas ${ }^{1}$, Silvaldo Felipe da Silveira ${ }^{1}$, Carlos Eduardo Pessanha da Silva Terra ${ }^{1} \&$ Messias Gonzaga \\ Pereira $^{2}$
}

${ }^{1}$ Laboratório de Entomologia e Fitopatologia; ${ }^{2}$ Laboratório de Melhoramento Genético Vegetal, Centro de Ciências Tecnológica e Agropecuária, Universidade Estadual do Norte Fluminense Darcy Ribeiro, 28013-600, Campos dos Goytacazes, RJ, Brasil

Autor para correspondência: Marcelo Vivas, e-mail: mrclvivas@hotmail.com

\begin{abstract}
RESUMO
Com o objetivo de se avaliar germoplasmas e híbridos de mamoeiro quanto à resistência a mancha-de-phoma em folhas, foram conduzidos três experimentos em blocos ao acaso com duas repetições na empresa Caliman Agrícola S/A, Linhares, Espírito Santo. Avaliou-se, em duas épocas, a incidência e a severidade da mancha-de-phoma em folhas de 50 genótipos e de 68 híbridos. Os genótipos que apresentaram menores severidades de doença foram 'Maradol', 'Maradol GL', 'Papaya 46', 'Tailândia' e 'SH 15-04' e os que apresentaram menores incidências foram 'Maradol', 'Maradol GL', 'Americano', 'Tailândia' e 'Baixinho de Santa Amália'. Os híbridos com menores incidências foram 'Americano x Waimanalo', 'Sekati x JS 12', 'Maradol x Taiwan et' e 'Maradol x Caliman G' enquanto que as menores severidades foram observadas em 'Calimosa x Tailândia', 'Calimosa x Maradol', 'Sekati x Caliman AM' e 'Sekati x JS 12'. Estes resultados orientarão o melhoramento voltado para a resistência à mancha-de-phoma na cultura do mamoeiro por meio de hibridizações, bem como auxiliarão na escolha de cultivares para o plantio, visando reduzir a necessidade de controle químico na cultura do mamoeiro.
\end{abstract}

Palavras-chave: Carica papaya, resistência genética.

\begin{abstract}
Reaction of germplasm and hybrid of papaya to phoma-spot (Phoma caricae-papayae) in field conditions

With the objective of evaluating germplasm and hybrids of papaya for their reaction to phoma-spot in leaves, three experiments were performed in a completely randomized block design with two replications at Agricultural Caliman S/A, in Linhares, Espírito Santo, Brazil. The incidence and severity of phoma-spot were evaluated in leaves of 50 genotypes and 68 hybrids. The lowest levels of disease severity were observed in 'Maradol', 'Maradol GL', 'Papaya 46', 'Tailândia', and 'SH 15-04' whereas the lowest incidences were detected in 'Maradol', 'Maradol GL', 'Americano', 'Tailândia' and 'Baixinho de Santa Amália'. The hybrids 'Calimosa x Tailândia', 'Calimosa x Maradol', 'Sekati x Caliman AM' and 'Sekati x JS 12' presented the lowest levels of severity, whereas 'Americano x Waimanalo', 'Sekati x JS 12', 'Maradol x Taiwan et' and 'Maradol x Caliman G' had the lowest incidence of disease. These results should be useful as a guide in breeding for resistance to phoma-spot of the papaya crop through hybridization, as well as in the recommendation of cultivars, seeking to reduce the necessity of chemical control in papaya crops.
\end{abstract}

Key words: Carica papaya, genetic resistance.

O mamoeiro (Carica papaya L.) é uma das fruteiras tropicais de maior importância no Brasil e no mundo, sendo cultivado em diversos estados brasileiros. Bahia e Espírito Santo são os principais estados produtores no Brasil, responsáveis por $88 \%$ da produção nacional, estimada em 1,9 milhões de toneladas em uma área de cultivo de 36,6 mil hectares (IBGE, 2008). Todavia, pouco do que se produz é exportado.

Entre os fatores responsáveis por esta limitada exportação estão as doenças, principalmente as que

Parte da Dissertação de Mestrado do primeiro autor. Universidade Estadual do Norte Fluminense Darcy Ribeiro. Campos dos Goytacazes RJ. 2009. ocorrem em pós-colheita. A podridão-peduncular é uma das principais doenças de pós-colheita. A doença é causada por um complexo de fungos, dentre os quais Phoma caricaepapayae, Colletotrichum gloesporioides, Lasiodiplodia theobromae, Alternaria alternata e Fusarium solani, são os mais comuns (Alvarez \& Nishijima, 1987; Rezende \& Francelli, 1997). A doença ocorre quando o fungo invade o fruto, após a colheita, através do pedúnculo cortado. Já foi constatada incidência de $100 \%$ de podridão-peduncular em frutos após a colheita (Liberato \& Costa, 1997), quando na ausência de quaisquer medidas de controle.

A mancha-de-phoma, causada por Phoma caricaepapayae (Tarr) Punith. (sin. Ascochyta caricae-papayae Tarr) é considerada a segunda doença mais importante em 
pós-colheita no Brasil (Rezende \& Francelli, 1997). O fungo é amplamente disseminado em regiões tropicais (Hunter e Budderhagen, 1972) e a doença apresenta sintomas variados. A mancha-de-phoma mostra-se de difícil controle nas áreas comerciais da Empresa Caliman Agrícola S/A, no município de Linhares, Espírito Santo. Nesta região a doença tem ocorrido nas folhas, o que contribui para aumentar o potencial de inóculo em campo. É notório que o controle do inóculo em campo, nas folhas, pode reduzir a incidência de podridão peduncular em pós-colheita. Suzuki et al. (2007) observaram ganhos de até $24 \%$ na redução de podridão-peduncular, quando foi efetuada a sanitização, com remoção das folhas senescentes e doentes. Portanto, há que se considerar que as folhas senescentes de mamoeiro podem servir como fonte de inóculo de $P$. caricae-papayae, para os frutos Desta forma o controle do patógeno nas folhas, terá reflexo na qualidade do fruto.

Como inexistem variedades resistentes, a pulverização de fungicidas tornou-se a principal medida de controle. Porém, o custo econômico, ambiental e social atribuído ao uso de agrotóxicos, bem como o surgimento de isolados de fitopatógenos resistentes a fungicidas no campo requerem pesquisas na busca de alternativas de controle de maior sustentabilidade, como o uso de cultivares geneticamente resistentes.

Embora não existam variedades resistentes, alguns trabalhos relatam a ocorrência de variabilidade entre os materiais avaliados quanto à resistência mancha-dephoma. Sanchez et al. (1991) avaliaram a reação à manchade-phoma em frutos de C. goudotiana, C. cauliflora (atualmente reclassificados no gênero Vasconcellea) e em duas cultivares de C. papaya ('Sunrise Solo 72/12' e 'Formoso') e mais quatro linhagens ('DCG-434(4pa)', 'DCG-422', 'DCG-435-5' e 'SS '). Os autores observaram resistência à mancha-de-phoma na espécie C. goudotiana em condições de campo e em pós-colheita.

Devido à limitação de informação a respeito da resistência genética e objetivando avaliar o potencial dos genótipos de mamão disponíveis na coleção de germoplasma UENF/Caliman quanto à resistência a mancha-de-phoma, conduziu-se três experimentos na Fazenda Macuco, da Empresa Caliman Agrícola S/A, localizada no município de Linhares, Estado do Espírito Santo, a $21 \mathrm{~m}$ de altitude. A região esta localizada na latitude de $19^{\circ} 15^{\prime}$ e longitude de $40^{\circ} 10^{\prime} \mathrm{com}$ precipitação pluvial média anual de $1100 \mathrm{~mm}$, temperatura média das máximas de $30,7^{\circ} \mathrm{C}$ a $34^{\circ} \mathrm{C}$ e temperatura média das mínimas de $15,8^{\circ} \mathrm{C}$ a $18,0^{\circ} \mathrm{C}$ (Suzuki et al., 2007).

Os três experimentos foram implantados em blocos ao acaso, com duas repetições, tendo cada repetição 20 plantas, dispostas em fileira dupla (10 plantas em cada fileira) no espaçamento 2,0 x 1,8 m. Entre tratamentos, lateralmente, adotou-se o espaçamento de 3,6 m. Sendo consideradas úteis nas avaliações as quatro plantas centrais de cada fileira.

O primeiro experimento correspondeu à avaliação da coleção de germoplasma UENF/Caliman, composta de
50 genótipos, sendo do grupo 'Solo' 19 genótipos: 'Caliman M5', 'Sunrise Solo 783', 'Taiwan et', 'Diva', 'Grampola', 'Sunrise Solo', 'Caliman AM', 'Caliman GB', 'Caliman SG', 'Caliman G', 'Sunrise Solo 72/12', 'Kapoho Solo PA', 'Baixinho de Santa Amália', 'Sunrise Solo TJ', 'São Mateus', 'Kapoho Solo PV', 'Sunrise Solo PT', 'Mamão Roxo' e 'Baixinho Super'; e do grupo 'Formosa' 31 genótipos: 'Costa Rica', 'Tailândia', 'Waimanalo', 'Mamão Bené', 'Maradol', 'Maradol GL', 'Sekati', 'Americano', 'STZ 51', 'STZ 52', 'Calimosa', 'JS 12', 'Cariflora', 'GTF', 'STZ 03', 'SH 12-07', 'SH 11-08', 'SH 50-09', 'SH 02-01', 'SH 14-05', 'SH 15-04', 'SH 04-02', 'SH 12-06', 'Papaya 42', 'Papaya 45', 'Papaya 46', 'FMV', 'Golden Robusto', 'Tainung', 'STZ 63' e 'Sekati FLM'.

$\mathrm{O}$ segundo experimento foi referente à avaliação de 22 híbridos $F_{1}$ originados de cruzamentos entre linhagens do grupo 'Solo' com o testador do grupo 'Formosa', 'JS 12', e de cruzamentos entre linhagens do grupo 'Formosa' com o testador do grupo 'Solo', 'Sunrise Solo 72/12', além do cruzamento entre 'Mamão Roxo x Sunrise Solo 72/12'. O terceiro experimento envolveu 46 híbridos $F_{1}$ provenientes de cruzamentos entre linhagens do grupo 'Solo' e 'Formosa' tendo como base para os cruzamentos os genótipos testadores 'Americano', 'Maradol' e 'Sekati', além dos cruzamentos do híbrido 'Calimosa' com os genótipos 'Tailândia', 'Maradol', 'Sekati' e 'Americano'.

A irrigação, do tipo microaspersão, foi suprida conforme as necessidades da cultura e os tratos culturais foram como recomendados para o mamoeiro (Marin et al., 1995). Ainda, por tratar-se de coleção de germoplasma e para se garantir produção de frutos e sementes, foram feitas pulverizações quinzenais de fungicidas registrados para a cultura. Nos 60 dias que antecederam a primeira avaliação, foram pulverizados os ingredientes ativos: pyraclostrobin (16/04, 23/04 e 21/05) e chlorothalonil (30/04). Antes da segunda avaliação, foram pulverizados: azoxystrobin (10/07 e 17/08), chlorothalonil (03/08), calda sulfocálcica $(10 / 08)$ e tiofanato metílico $(27 / 07)$.

Foram realizadas duas avaliações, sendo a primeira no final de maio e a segunda no final de agosto de 2007. Avaliou-se a incidência e a severidade de mancha-dephoma em folhas de mamoeiro. A incidência de folhas com sintomas de mancha-de-phoma foi obtida em percentagem pela relação entre o número de folhas com sintomas pelo número total de folhas. Para severidade de mancha-dephoma avaliou-se a folha disposta imediatamente abaixo da folha com a primeira flor aberta, com o auxílio de escala diagramática proposta por Terra et al. (2008).

Para cada doença foram conduzidas análises de variâncias conjuntas, considerando época de avaliação como fonte de variação. As análises de variâncias foram realizadas utilizando-se o Programa SAS (SAS, 1992) e as médias dos genótipos foram comparadas pelo teste de agrupamento de Scott-Knott, a 0,05 de probabilidade utilizando-se o aplicativo SAEG (Euclides, 1983). 
Reação de germoplasma e híbridos de mamoeiro à mancha-de-phoma...

Com base na incidência de folhas com sintoma de mancha-de-phoma, avaliada no germoplasma (experimento
1) houve a formação de cinco grupos (Tabela 1). Desta forma estabeleceu-se a seguinte classificação: genótipos

TABELA 1 - Médias de incidência e severidade de mancha-de-phoma em folhas de genótipos (germoplasma) de mamoeiro pertencentes à coleção de germoplasma da UENF/CALIMAN, avaliadas em Linhares ES, no ano de 2007

\begin{tabular}{|c|c|c|c|c|}
\hline \multirow{2}{*}{$\begin{array}{l}\text { Genótipos (Germoplasma) } \\
\text { 'Maradol' }\end{array}$} & \multicolumn{2}{|c|}{ Incidência } & \multicolumn{2}{|c|}{ Severidade } \\
\hline & 30,876 & $\mathrm{E}^{*}$ & 0,842 & $\mathrm{~B}$ \\
\hline 'Maradol GL' & 50,681 & $\mathrm{D}$ & 1,384 & $\mathrm{~B}$ \\
\hline ‘Americano' & 50,797 & $\mathrm{D}$ & 5,400 & $\mathrm{~B}$ \\
\hline 'Tailândia' & 59,751 & $\mathrm{C}$ & 2,592 & $\mathrm{~B}$ \\
\hline 'Baixinho de Santa Amália' & 61,244 & $\mathrm{C}$ & 4,450 & $\mathrm{~B}$ \\
\hline 'JS 12 ' & 62,428 & $\mathrm{C}$ & 3,888 & $\mathrm{~B}$ \\
\hline 'Tainung' & 62,729 & $\mathrm{~B}$ & 5,050 & $\mathrm{~B}$ \\
\hline 'Waimanalo' & 62,941 & $\mathrm{C}$ & 6,363 & $\mathrm{~B}$ \\
\hline 'Sekati' & 63,524 & $\mathrm{C}$ & 4,444 & $\mathrm{~B}$ \\
\hline 'Cariflora' & 66,612 & $\mathrm{~B}$ & 4,202 & $\mathrm{~B}$ \\
\hline 'Mamão Bené' & 66,820 & $\mathrm{~B}$ & 5,000 & $\mathrm{~B}$ \\
\hline 'Baixinho Super' & 68,014 & $\mathrm{~B}$ & 5,250 & $\mathrm{~B}$ \\
\hline 'SH $50-09$ ' & 68,201 & $\mathrm{~B}$ & 4,794 & $\mathrm{~B}$ \\
\hline 'SH $12-06$ ' & 68,288 & $\mathrm{~B}$ & 5,594 & $\mathrm{~B}$ \\
\hline 'SH $02-01$ ', & 68,467 & $\mathrm{~B}$ & 6,113 & $\mathrm{~B}$ \\
\hline 'Calimosa' & 69,339 & $\mathrm{~B}$ & 4,438 & $\mathrm{~B}$ \\
\hline 'Grampola' & 69,654 & $\mathrm{~B}$ & 8,281 & $\mathrm{~B}$ \\
\hline 'SH $04-02$ ' & 69,895 & $\mathrm{~B}$ & 6,531 & $\mathrm{~B}$ \\
\hline 'Caliman M5' & 71,980 & $\mathrm{~B}$ & 7,056 & B \\
\hline 'SH $14-05$ ' & 72,006 & $\mathrm{~B}$ & 5,550 & $\mathrm{~B}$ \\
\hline 'SH $12-07$ ' & 72,391 & $\mathrm{~B}$ & 4,206 & $\mathrm{~B}$ \\
\hline 'STZ 03' & 73,445 & $\mathrm{~B}$ & 6,694 & $\mathrm{~B}$ \\
\hline 'SH $15-04$ ' & 73,768 & $\mathrm{~B}$ & 3,656 & $\mathrm{~B}$ \\
\hline 'Papaya 42' & 74,643 & $\mathrm{~A}$ & 6,625 & $\mathrm{~B}$ \\
\hline 'Costa Rica' & 74,864 & $\mathrm{~A}$ & 6,294 & $\mathrm{~B}$ \\
\hline 'Taiwan et' & 75,751 & $\mathrm{~A}$ & 5,263 & $\mathrm{~B}$ \\
\hline 'FMF' & 75,880 & $\mathrm{~A}$ & 6,506 & $\mathrm{~B}$ \\
\hline 'Sekati FLM' & 75,925 & $\mathrm{~A}$ & 5,490 & $\mathrm{~B}$ \\
\hline 'Sunrise Solo 72/12' & 75,933 & $\mathrm{~A}$ & 5,838 & $\mathrm{~B}$ \\
\hline 'STZ 51' & 76,191 & $\mathrm{~A}$ & 8,094 & $\mathrm{~A}$ \\
\hline 'Sunrise Solo 783’ & 76,365 & $\mathrm{~A}$ & 11,950 & $\mathrm{~A}$ \\
\hline 'Sunrise Solo' & 76,607 & $\mathrm{~A}$ & 7,569 & $\mathrm{~A}$ \\
\hline 'Diva' & 77,140 & $\mathrm{~A}$ & 5,163 & $\mathrm{~B}$ \\
\hline 'STZ 63' & 77,641 & $\mathrm{~A}$ & 8,600 & $\mathrm{~A}$ \\
\hline 'SH $11-08$ ' & 78,017 & $\mathrm{~A}$ & 5,044 & $\mathrm{~B}$ \\
\hline 'GTF' & 78,032 & $\mathrm{~A}$ & 8,092 & $\mathrm{~A}$ \\
\hline 'Caliman G' & 78,044 & A & 8,675 & $\mathrm{~A}$ \\
\hline 'Papaya 45' & 78,090 & A & 4,634 & B \\
\hline 'Sunrise Solo TJ' & 78,460 & $\mathrm{~A}$ & 5,688 & $\mathrm{~B}$ \\
\hline 'Kapoho Solo PV' & 78,824 & A & 6,669 & $\mathrm{~B}$ \\
\hline 'STZ 52' & 79,020 & A & 7,019 & $\mathrm{~B}$ \\
\hline 'Kapoho Solo PA' & 79,640 & A & 8,819 & $\mathrm{~A}$ \\
\hline 'Mamão Roxo' & 80,060 & $\mathrm{~A}$ & 6,894 & $\mathrm{~B}$ \\
\hline 'Papaya 46’ & 80,092 & $\mathrm{~A}$ & 2,413 & $\mathrm{~B}$ \\
\hline 'Caliman SG' & 80,461 & A & 15,188 & $\mathrm{~A}$ \\
\hline 'Caliman GB' & 80,829 & $\mathrm{~A}$ & 10,125 & $\mathrm{~A}$ \\
\hline ‘São Mateus' & 82,120 & $\mathrm{~A}$ & 4,000 & $\mathrm{~B}$ \\
\hline 'Sunrise Solo PT' & 82,564 & $\mathrm{~A}$ & 5,900 & $\mathrm{~B}$ \\
\hline 'Golden robusto' & 82,874 & $\mathrm{~A}$ & 11,469 & $\mathrm{~A}$ \\
\hline 'Caliman AM' & 83,661 & $\mathrm{~A}$ & 11,313 & $\mathrm{~A}$ \\
\hline
\end{tabular}

*Na mesma coluna, médias seguidas de mesma letra não diferem entre si pelo teste Scott-knott em nível de 5\% de probabilidade. 
locados no primeiro e segundo grupo foram considerados resistentes, no terceiro moderadamente resistentes e os demais suscetíveis. O primeiro grupo, formado apenas pelo genótipo 'Maradol'. Os genótipos 'Maradol GL' e 'Americano' ficaram locados no segundo grupo. No terceiro grupo ficaram os genótipos 'Tailândia', 'Baixinho de Santa Amália', 'JS 12', 'Waimanalo' e 'Sekati'. Os demais genótipos foram distribuídos nos dois grupos restantes.

Quando se analisou a severidade de mancha-dephoma, observou-se a formação de dois grupos sendo o grupo com os menores valores médios formado por 37 genótipos (Tabela 1). Entre os genótipos com menores severidades de mancha-de-phoma encontramse 'Maradol', 'Maradol GL', 'Americano', bem como 'Tailândia', 'Baixinho de Santa Amália', 'JS 12', 'Waimanalo' e 'Sekati', considerados, respectivamente, resistentes e moderadamente resistentes para a incidência de folhas com sintoma de mancha-de-phoma. Estes resultados apontam tais genótipos como promissores para a obtenção de cultivares como maiores níveis de resistência a mancha-de-phoma.

Trabalhos anteriores relatam a ocorrência de variabilidade destes materiais para outras doenças. Ide et al. (2001) destacaram a cultivar 'Maradol' como resistente, por apresentar menor número de pinta-preta no lóbulo central em relação a variedade 'Sunrise Solo 72/12'. Dianese et al. (2007) relataram que o genótipo 'Sekati' foi o que apresentou menor severidade de pintapreta nas folhas. Santos et al (2009) apontaram o genótipo 'Tailândia' como resistente à oídio por apresentar, em casa de vegetação, a menor de incidência de folhas de mamoeiro com sintoma desta doença. Desta forma, diante do exposto, é possível a obtenção de linhagens e, ou híbridos com resistência múltipla a doenças, uma vez que os genótipos 'Maradol' e 'Sekati' apresentam certo grau de resistência a pinta-preta e a mancha-de-phoma, e 'Tailândia' resistência a oídio e a mancha-de-phoma.

Nas avaliações de incidência de mancha-de-phoma realizadas nas combinações híbridas envolvendo os genitores 'JS 12' e 'Sunrise Solo 72/12' (experimento 02), não foi possível observar efeito de nenhum dos cruzamentos na redução de incidência, sendo que a média para estes cruzamentos variou de 75,315 (no híbrido 'Sunrise Solo 72/12 x Maradol GL') a 83,631 (no híbrido 'Sunrise Solo 72/12 x Sekati'). Já para severidade houve a formação de dois grupos, sendo o grupo mais resistente, composto por 13 combinações. Das combinações com o genótipo 'JS 12', as menores médias ocorreram quando este foi combinado com 'Sunrise Solo PT', 'Sunrise Solo', 'Caliman M5', 'Grampola', 'São Mateus', 'Kaphoro Solo PV', 'Caliman GB', 'Baixinho de Santa Amália' e 'Diva'. Já para o genótipo 'Sunrise Solo 72/12' as melhores combinações foram com 'Maradol', 'Maradol GL', 'Sekati' e 'Tailândia'. Por estes resultados, constata-se que os genótipos 'Maradol', 'Maradol GL', 'Tailândia', 'Baixinho de Santa Amália', 'Sekati' e 'São Mateus', além de apresentarem resistência à mancha-de-phoma, também podem ser utilizados em cruzamentos visando à redução da doença em híbridos que agreguem características como resistência à doença supra citada, bem como atributos morfoagronômicas importantes.

Considerando a incidência de folhas com mancha-de-phoma, avaliadas nos híbridos contidos no terceiro experimento, houve a formação de três grupos, as combinações 'Americano x Waimanalo' e 'Maradol x JS 12' estiveram, respectivamente, locadas nos dois grupos mais resistente (Tabela 2). Com base na avaliação da severidade da mancha-de-phoma, neste experimento, deve-se destacar os híbridos 'Calimosa x Sekati', 'Calimosa x Maradol' e 'Sekati x Caliman AM', com severidade de doença inferior a 2\% (Tabela 2). Considerando as melhores combinações envolvendo cada genótipo testador em particular, destacam-se, as combinações 'Americano x Waimanalo' e 'Americano x São Mateus', ambas com menores severidades de mancha-de-phoma para o testador 'Americano'. Para o genótipo 'Maradol' todos os cruzamentos resultaram em híbridos resistentes. Já para o genótipo 'Sekati', as melhores combinações ocorreram com 'Caliman SG', 'Caliman G', 'Diva', 'JS 11', 'Sunrise Solo', 'Caliman GB' e 'Caliman AM'.

Cattaneo (2001), trabalhando com marcadores RAPD e AFLP, para estudar a diversidade genética de 22 genótipos de mamoeiro, agrupou os genótipos 'Caliman G', 'Caliman SG', 'Caliman GB', 'Grampola', 'Caliman D' e 'Sunrise Solo TJ' em um mesmo grupo. Portanto, sugere-se que 'Caliman SG', 'Caliman G' e 'Caliman GB' portam genes comuns, uma vez que se comportaram de forma semelhante quando combinadas com os genótipos 'Americano', 'Maradol' e 'Sekati'. No entanto, quando se analisa o comportamento dos genótipos isoladamente observa-se que estes ficaram no grupo de maior severidade da doença. De forma semelhante, as combinações destes genótipos com o testador 'Americano' se posicionaram novamente dentro do grupo de maior severidade (Tabela 2). Assim, analisando os genótipos isoladamente e em cruzamento denota-se que os portadores do gene de resistência são os genótipos 'Maradol' e 'Sekati'.

Com base nos dados de incidência e severidade de doença obtidas nos experimentos 02 e 03, foi possível identificar como cada material se comporta como doador, passando a característica desejável a seus descendentes. Assim, ressaltam-se os genótipos 'Americano', 'Waimanalo', 'Sekati', 'JS 12', 'Maradol', 'Maradol GL', 'Tailândia', 'Baixinho de Santa Amália' e 'São Mateus', como possíveis portadores de alelos que tendem a contribuir para a redução de mancha-de-phoma em híbridos de mamoeiro. 
Reação de germoplasma e híbridos de mamoeiro à mancha-de-phoma...

TABELA 2 - Médias de incidência e severidade de mancha-de-phoma em folhas de híbridos de mamoeiro derivados dos genótipos testadores 'Americano', 'Maradol' e 'Sekati', avaliadas em Linhares ES no ano de 2007

\begin{tabular}{|c|c|c|c|c|}
\hline \multirow{2}{*}{$\begin{array}{l}\text { Genótipos (Híbridos) } \\
\text { 'Americano x Waimanalo' }\end{array}$} & \multicolumn{2}{|c|}{ Incidência } & \multicolumn{2}{|c|}{ Severidade } \\
\hline & 54,299 & $\mathrm{C}^{*}$ & 2,906 & $\mathrm{~B}$ \\
\hline 'Maradol x JS 12' & 67,320 & $\mathrm{~B}$ & 2,319 & $\mathrm{~B}$ \\
\hline 'Sekati x JS 11’' & 70,951 & A & 3,438 & $\mathrm{~B}$ \\
\hline 'Maradol x Caliman G' & 73,231 & A & 2,656 & $\mathrm{~B}$ \\
\hline ‘Calimosa x Maradol’' & 73,349 & A & 1,292 & $\mathrm{~B}$ \\
\hline 'Americano x Sekati' & 73,504 & A & 7,750 & $\mathrm{~A}$ \\
\hline 'Sekati x JS 12’ & 74,945 & A & 5,069 & A \\
\hline 'Americano x Baixinho de Santa Amália' & 75,370 & A & 5,219 & $\mathrm{~A}$ \\
\hline 'Americano x Baixinho Super' & 76,096 & A & 6,844 & $\mathrm{~A}$ \\
\hline 'Calimosa x Americano' & 76,192 & A & 4,975 & A \\
\hline 'Sekati x Caliman G' & 76,558 & A & 4,013 & $\mathrm{~B}$ \\
\hline 'Americano x São Mateus' & 76,893 & A & 4,769 & $\mathrm{~B}$ \\
\hline 'Sekati x Caliman GB' & 77,701 & A & 4,681 & $\mathrm{~B}$ \\
\hline 'Sekati x Caliman M5' & 77,755 & A & 5,356 & A \\
\hline 'Maradol x Sunrise Solo 72/ 12' & 77,796 & A & 4,344 & $\mathrm{~B}$ \\
\hline 'Americano x Grampola' & 77,846 & $\mathrm{~A}$ & 9,063 & $\mathrm{~A}$ \\
\hline 'Americano x Sunrise Solo PT' & 77,879 & A & 6,613 & A \\
\hline 'Maradol x Sunrise Solo' & 77,887 & A & 3,931 & $\mathrm{~B}$ \\
\hline 'Calimosa x Sekati' & 77,906 & A & 4,375 & $\mathrm{~B}$ \\
\hline 'Sekati x Sunrise Solo' & 78,209 & $\mathrm{~A}$ & 4,638 & $\mathrm{~B}$ \\
\hline 'Calimosa x Tailândia' & 78,468 & $\mathrm{~A}$ & 1,208 & $\mathrm{~B}$ \\
\hline ‘Sekati x Caliman SG’ & 78,730 & A & 3,719 & $\mathrm{~B}$ \\
\hline ‘Americano x Mamão Roxo' & 78,749 & A & 5,081 & A \\
\hline 'Americano x STZ 52' & 78,771 & A & 6,394 & A \\
\hline 'Americano x Diva' & 79,073 & A & 7,356 & $\mathrm{~A}$ \\
\hline 'Maradol x Caliman SG' & 79,166 & A & 2,894 & $\mathrm{~B}$ \\
\hline 'Taiwan et x Americano' & 79,213 & A & 5,669 & $\mathrm{~A}$ \\
\hline 'Americano x Kapoho Solo PV' & 79,314 & A & 8,344 & A \\
\hline 'Americano x Caliman SG' & 79,558 & A & 7,613 & A \\
\hline 'Sekati x Diva' & 79,776 & A & 4,031 & $\mathrm{~B}$ \\
\hline 'Americano x Caliman M5' & 79,804 & A & 5,394 & $\mathrm{~A}$ \\
\hline 'Sunrise Solo 72/ 12 x Americano' & 79,873 & A & 6,575 & $\mathrm{~A}$ \\
\hline 'Sekati x Caliman AM' & 80,134 & A & 1,850 & $\mathrm{~B}$ \\
\hline 'Sunrise Solo 72/ 12 x Sekati' & 80,739 & A & 4,456 & $\mathrm{~B}$ \\
\hline 'Maradol x Caliman AM' & 80,751 & $\mathrm{~A}$ & 3,100 & $\mathrm{~B}$ \\
\hline 'Americano x Caliman GB' & 80,922 & A & 7,444 & A \\
\hline 'Americano x Caliman AM' & 81,169 & A & 5,150 & $\mathrm{~A}$ \\
\hline 'Americano x Caliman G' & 81,726 & A & 7,313 & A \\
\hline 'Americano x Costa Rica' & 82,581 & $\mathrm{~A}$ & 5,875 & A \\
\hline 'Maradol x Sunrise Solo PT' & 82,663 & A & 3,288 & $\mathrm{~B}$ \\
\hline 'Maradol x Caliman M5' & 82,739 & A & 3,531 & $\mathrm{~B}$ \\
\hline 'Americano x Sunrise Solo' & 83,376 & A & 5,363 & A \\
\hline 'Maradol x Diva' & 83,544 & A & 3,719 & $\mathrm{~B}$ \\
\hline 'Americano x Sunrise Solo 783' & 83,733 & A & 5,400 & A \\
\hline 'Americano x Sunrise Solo TJ' & 84,607 & A & 6,225 & A \\
\hline
\end{tabular}

*Na mesma coluna, médias seguidas de mesma letra não diferem entre si pelo teste Scott-knott em nível de 5\% de probabilidade.

\section{REFERÊNCIAS BIBLIOGRÁFICAS}

Alvarez AM, Nishijima WT (1987) Postharvest diseases of papaya. Plant Disease 71:681-686.

Cattaneo LF (2001) Avaliação da divergência genética e análise de gerações em mamoeiro (Carica papaya L.). Tese de Doutorado. Universidade Estadual do Norte Fluminense - UENF. Campos dos Goytacazes RJ.

Dianese AC, Blum LEB, Dutro JB, Lopes LF, Sena MC, Freitas LF, Yamanishi OK (2007) Reação de genótipos de mamoeiro à varíola e à podridão-do-pé. Fitopatologia Brasileira 32:419-423.

Euclides RF (1983) Sistema para análises estatísticas e genéticas 
(SAEG). Divisão de pesquisa e desenvolvimento/CPD. Viçosa MG. Universidade Federal de Viçosa.

Hunter JE, Buddenhagen IW (1972) Incidence, epidemiology and control of fruit diseases of papaya in Hawaii. Tropical Agriculture 46:61-71.

IBGE (2008) - Instituto Brasileiro de Geografia e Estatística. Produção Agrícola Municipal. Rio de Janeiro RJ. Disponível em: $<\mathrm{http}$ ://www.sidra.ibge.gov.br $>$. Acesso em 5 março de 2008.

Ide CD, Martelleto LAP, Marin SLD, Yamanishi OK (2001) Resposta de diferentes genótipos de mamoeiro à manifestação de varíola. PESAGRO-RIO, Niterói RJ. Comunicado Técnico n ${ }^{\circ} 259$.

Liberato JR, Costa H (1997) Incidência de antracnose e podridão peduncular em frutos de mamoeiro em Linhares, ES. Fitopatologia Brasileira 22:276 (Resumo)

Marin SLD, Gomes JA, Salgado JS, Martins DS, Fullin EA (1995) Recomendações para a cultura do mamoeiro dos grupos Solo e Formosa no Estado do Espírito Santo. 4 ed. Vitória ES. EMCAPA, Circular Técnica 3.

Rezende JAM, Fancelli MI (1997) Doenças do mamoeiro (Carica papaya L.). In: Kimati H, Amorim L, Bergamin Filho A, Camargo LEA, Rezende JAM (Eds.) Manual de Fitopatologia. Doenças das plantas cultivadas. São Paulo SP. Agronômica Ceres. pp. 486496.

Sanchez M, Dianese JC, Costa CL (1991) Fatores determinantes do dano de Phoma caricae-papayae ao fruto do mamoeiro (Carica papaya) e detecção de resistência ao fungo em Carica gaudotiana. Fitopatologia Brasileira 16:121-129.

Santos PHD, Vivas M, Terra CEPS, Silveira SF (2009) Avaliação de produtos alternativos no controle de oídio (Oidium caricae) em genótipos de mamoeiro. In: Costa AFS, Costa AN (Orgs.) $4^{\circ}$ Simpósio do Papaya Brasileiro. Vitória ES, Incaper. Documentos, CD-ROM 013.

SAS Institute INC. (1992) Statistical Analysis System. Release 6.12 (Software).

Suzuki MS, Zambolim L, Liberato JR (2007) Progresso de doenças fúngicas e correlação com variáveis climáticas em mamoeiro. Summa Phytopathologica 33:167-177.

Terra CEPS, Vivas M, Melo CS, Silveira SF, Pereira MG (2008) Elaboração e validação de escala diagramática para avaliação da severidade da podridão preta (Phoma caricae papayae) em folhas de mamoeiro (Carica papaya). Anais, XX Congresso Brasileiro de Fruticultura, Vitória ES. 